Infant weaning

\section{Introduction of solids to the infant diet}

\section{G A Khakoo, G Lack}

\section{Commentary on the papers by Morgan et al and Zutavern et al}

r he Department of Health has recently supported the WHO advice for mothers to exclusively breast feed until 6 months of age. ${ }^{1}$ Furthermore, there are expert committee guidelines regarding the introduction of solids in the diet of infants at high risk of developing allergy. The joint guidelines of the European Society for Paediatric Allergology and Clinical Immunology (ESPACI) and the European Society for Paediatric Gastroenterology, Hepatology and Nutrition (ESPGHAN) recommend that solid food introduction be delayed until 5 months of age. The American Academy of Pediatrics advice is more detailed, suggesting that solids be delayed until 6 months of age, cows' milk to 1 year, egg to 2 years, and peanuts, tree nuts, and fish to 3 years. ${ }^{2}$ It is not surprising that these guidelines differ as they are based on the limited evidence of two studies. The first, a prospective non-randomised study in an atopic population, suggested that eczema and a history of food allergy was reduced at age 1 year in infants fed solids after 6 months of age compared with those infants with solids introduced at 3 months, but no difference was seen at 5 year follow up. ${ }^{3}$ The second study had a similar design, but was conducted in a normal population. This study showed more eczema, but not asthma, at 2-4 years of age in infants fed four or more solid foods before age 4 months compared with infants receiving no solid foods before 4 months of age. This difference was maintained until 10 years of age. ${ }^{4}$

The increased information to health professionals and the public regarding dietary interventions which are perceived to reduce allergy has resulted in the risk of recent dietary intervention studies being confounded by reverse causality. Thus, mothers who are atopic, come from atopic families, or have a baby already showing signs of allergy, for example, eczema, may take steps that they perceive will reduce the chance of their children developing allergies, or be advised by health profes-

The addition of two studies, published in this issue, looking at the effect on allergy of the timing of introduction of solids to the infant $\operatorname{diet}^{56}$ is most welcome in view of the limited literature to date. The study of Morgan et al prospectively followed up 257 well preterm infants from three maternity units, with follow up to 1 year of age. The introduction of four or more solid foods before 17 weeks post-term was associated with a higher risk of eczema in infants with and without a family history of allergy. ${ }^{5}$ Unfortunately no data are presented on the extent of prematurity, which may confound the results since increasing prematurity is known to be associated with a decreased risk of atopy. ${ }^{7}$ This could be due to a higher infectious burden and altered gut microbial flora in more preterm infants, with higher risks of sepsis and necrotising enterocolitis. Such infants would also be more likely to have delayed introduction of solids. Thus the observed relation between early introduction of solids and infant atopy could be confounded by the higher infectious burden in more premature infants in whom solids are delayed for medical reasons.

The study of Zutavern et al is a well conducted prospective birth cohort study involving 642 infants, with follow up to $5 \frac{1}{2}$ years of age. ${ }^{6}$ The dietary data were collected prospectively, the allergic phenotype was carefully documented for wheeze and eczema, and objective skin prick test data were also collected. The data were corrected for parental asthma and atopy. This study shows no evidence to support a protective effect of sionals to do so. late introduction of solids on the development of eczema or asthma. The authors mention that the increased risk of eczema associated with the late introduction of egg is likely to be due to reverse causality.

In summary, the study of Morgan et al suggests that in preterm infants a delay in the introduction of solids may help to reduce the development of early childhood allergy. However, in the general population, the study of Zutavern et al challenges the widely held belief that delayed introduction of solids reduces the risk of allergy. These studies add to the current conflicting literature, which taken as a whole does not allow an authoritative statement to be made regarding the relation between the introduction of solids and the development of allergy. Future studies will need to take into account confounding factors such as reverse causality, and identify specific endpoints, such as food allergy, eczema, and asthma.

Arch Dis Child 2004:89:295.

doi: 10.1136/adc.2003.039016

\section{Authors' affiliations}

G A Khakoo, G Lack, Department of Paediatric Allergy, Asthma and Immunology, Imperial College London at St Mary's Hospital, London, UK

Correspondence to: $\operatorname{Dr} G$ Lack, Department of Paediatric Allergy, Asthma and Immunology, Imperial College London at St Mary's Hospital, Salton House, St Mary's Hospital, Praed Street, London W2 iNY, UK; gideon.lack@ st-marys.nhs.uk

\section{REFERENCES}

1 Foote KD, Marriott LD. Weaning of infants. Arch Dis Child 2003:88:488-92.

2 Zeiger RS. Food allergen avoidance in the prevention of food allergy in infants and children. Pediatrics 2003;111:1662-9.

3 Kajosaari M. Atopy prophylaxis in high-risk infants: prospective 5-year follow-up of children with six months exclusive breast feeding and solid food elimination. Adv Exp Med Biol 1991;310:453-8.

4 Fergusson DM, Horwood U, Shannon FT. Early solid feeding and recurrent childhood eczema: a 10-year longitudinal study. Pediatrics 1990;86:541-6.

5 Morgan J, Williams P, Norris F, et al. Eczema and early solid feeding in preterm infants. Arch Dis Child 2004;89:309-14

6 Zutavern A, von Mutius E, Harris J, et al. The introduction of solids in relation to asthma and eczema. Arch Dis Child 2004;89:303-8.

7 Siltanen $M$, Kajosaari $M$, Pohjavuori $M$, et al. Prematurity at birth reduces the long-term risk of atopy. J Allergy Clin Immunol 2001;107:229-34. 
Growth

\section{Height monitoring as a diagnostic test}

\section{P C Hindmarsh, T J Cole}

\section{Commentary on the paper by van Buuren et al}

M easurement of height is an important component of child health care and has been widely incorporated into paediatric practice. Yet little is known about how it performs in terms of sensitivity and specificity for detecting growth disorders. This lack of information impacts on health care in a number of ways. First, it is difficult to inform public health policy via recommendations for height monitoring, which has resulted in a plethora of statements made about referral for height assessment. One consequence of this has been to opt for a minimum standard for practice as exemplified in Health for all children. ${ }^{1}$ Second, the lack of information on test performance in the early steps of the short stature evaluation decision tree makes it difficult to interpret subsequent tests and ultimately the likelihood of the presence or absence of disease. ${ }^{2}$

The Dutch study reported by van Buuren and colleagues in this issue ${ }^{3}$ addresses for the first time these issues of test performance by quantifying the role of height monitoring in the identification of girls with Turner's syndrome (TS). TS is the ideal condition to use to show the methodology as it fulfils several important screening criteria-it is common ( 1 in 2500 live female births), a confirmatory test is available with high sensitivity and specificity (karyotype), and early intervention can appreciably influence outcome (growth, osteoporosis, and management of ovarian dysfunction). One of the problems with TS is that universal karyotype screening is unfeasibly expensive-a pre-karyotype assessment is required. The clinical manifestations of TS are variable whereas the short stature, particularly with respect to parental height, is not, so height monitoring clearly should play an important role.

The Dutch group treats height monitoring as a diagnostic test using two distinct populations, TS girls (cases) and normal girls (controls), which together provide estimates of sensitivity, specificity, and median referral age for a series of distinct screening rules for referral for height assessment. The three basic rules they consider, which are based on Dutch guidelines, are: (1) height standard deviation score (SDS) below a given cut-off; (2) height SDS below a given cut-off based on target height; and (3) height SDS velocity below a given cutoff. The performance of these rules, both separately and in combination, is assessed for a series of distinct cut-offs and age(s) when they apply, and the best performing rules identified.

What should we look for in a screening rule? It needs a high sensitivity, so it identifies most girls with TS, but more importantly it must have a very low false positive rate (that is, very high specificity). A false positive rate exceeding say $1 \%$ (specificity <99\%) would have serious implications for the workload of specialist growth clinics. With this in mind the British 1990 height reference chart ${ }^{4}$ includes a 0.4 th centile curve which predicts a false positive rate of only about $0.4 \%,{ }^{5}$ corresponding to an absolute height SDS rule with a cut-off of -2.67 .

With this in mind the results of the Dutch study are enlightening. The absolute height rule performs relatively poorly, with a specificity of only $98.1 \%$ (sensitivity $41 \%$ ) with a cut-off of -3.5 up to age 3 and 3.0 afterwards. This is appreciably worse than the $99.9 \%$ predicted theoretically, and the reason why it performs so poorly is not obvious. It may be because the Dutch height reference does not adjust birth length for gestation.

The parentally adjusted rule has a much higher specificity, up to $99.4 \%$ or better, and its sensitivity is also higher, near $70 \%$. The deflection (velocity) rule gives specificities close to $100 \%$ but sensitivities below 60\%. The authors propose a combined rule involving these two components with specificity $99.4 \%$ and sensitivity $79 \%$.

Two strengths of the approach are the ability to compare the performance of different screening rules, and the use of pre-existing data. This means that large prospective studies are not required, and that screening rules can be developed for any growth disorder where suitable data exist. For the purists, one slight disadvantage is that the estimates of sensitivity and specificity are potentially biased. This is because the TS population is itself biased, consisting of girls who have had to draw attention to themselves to be identified. We do not know what proportion of TS patients were missed in assembling the TS cohort. If the factor identifying TS girls was short stature, this might improve test performance. Also, using datasets drawn over a long period of time may tend to incorporate the more severely affected in the earlier years. As a result the sensitivity and specificity results need to be interpreted with caution.

The clinical significance of the findings is intriguing. First, the current UK view is that height velocity does not contribute usefully to growth monitoring, ${ }^{16}$ yet one of the proposed screening rules includes height velocity. Second, the findings confirm the value of parental height adjustment. So how should these results affect the UK recommendations for height assessment? Measuring height velocity involves two sets of costs: the resource cost of having to collect the longitudinal height data, and the delay cost of potential cases having to wait an extra year or more before being diagnosed, rather that relying on their height at presentation. So does the benefit of including height velocity justify the cost? In our view the answer is no. Adding velocity to the parentally adjusted rule with cut-off -2 increases the sensitivity by just 3\% for the same specificity. A better approach would be to focus on the parental height rule, which can in theory be improved using formal regression methods-that is, height adjusted for familial height. ${ }^{78}$ The authors' methodology could quantify the benefit of this approach.

From the epidemiologist's standpoint these results are valuable in showing how to study the performance of growth assessment techniques "in the field". The ideal approach would be to compare height measurement performance with a karyotype assessment in all girls born in the UK, but such a study would be very expensive-only 120-150 TS girls are born each year, so the study would need to last several years. However, if one accepts that the sensitivity and specificity may be different in the "field", then at least the proposed approach allows for a more precise estimate of the role of height monitoring in the population, and provides a methodology which could be applied to other areas of interest such as growth hormone deficiency. The approach and the information it provides are to be welcomed and should now be used to inform height monitoring practice.

Arch Dis Child 2004;89:296-297. doi: 10.1136/adc.2003.039560 


\section{Authors' affiliations}

P C Hindmarsh, Centre for Human Growth and Maturation, Institute of Child Health, University College London, London, UK T J Cole, Centre for Paediatric Epidemiology and Biostatistics, Institute of Child Health, University College London, London, UK

Correspondence to: Dr P C Hindmarsh, The Middlesex Hospital, Mortimer Street, London WIN 8AA, UK; p.hindmarsh@ucl.ac.uk

\section{REFERENCES}

1 Hall DMB. Health for all children, 4th edn Oxford: Oxford University Press, 2003.

2 Sox HC, Blatt MA, Higgins MC, et al. Medical decision making. Boston: Butterworth-Heinemann, 1988.

3 van Buuren S, van Dommelen P, Zandwijken GRJ, et al. Towards evidence based referral criteria for growth monitoring. Arch Dis Child 2004;89:336-41.

4 Freeman JV, Cole TJ, Chinn S, et al. Cross sectional stature and weight reference curves for the UK, 1990. Arch Dis Child 1995;73:17-24

5 Cole TJ. Do growth chart centiles need a face lift? BMJ 1994;308:641-2.

6 Voss LD, Wilkin TJ, Bailey BJR, et al. The reliability of height and height velocity in the assessment of growth (the Wessex Growth Study). Arch Dis Child 1991;66:833-7.

7 Cole TJ. A simple chart to assess non-familial short stature. Arch Dis Child 2000;82:173-6.

8 Hermanussen M, Cole TJ. The calculation of target height reconsidered. Horm Res 2003;59:180-3.
Infection

\section{MRSA: the problem reaches paediatrics}

\section{J W Gray}

\section{Commentary on the paper by Khairulddin et al}

7 , he pattern of MRSA in UK hospitals nowadays is very different to that seen a decade or so ago. Then, MRSA was confined mainly to a relatively small number of hospitals in the southeast of England and some of the large provincial conurbations. ${ }^{1}$ However, new strains of epidemic MRSA, especially EMRSA-15 and EMRSA-16, have since emerged and spread to become established to some extent in virtually every hospital in the country. ${ }^{1}$ Between 1992 and 2002 the proportion of blood culture isolates of Staphylococcus aureus reported by microbiology laboratories to the Communicable Disease Surveillance Centre that were methicillin resistant increased from 3\% to $43 \%{ }^{2}$ The pervasiveness of MRSA is underlined by the fact that MRSA now accounts for over $30 \%$ of $S$ aureus bacteraemias in every health care region in England, Wales, and Northern Ireland. ${ }^{3}$

MRSA are frequently not only resistant to methicillin and other $\beta$-lactam antibiotics, but to other classes of antibiotics as well. ${ }^{1}$ The glycopeptide antibiotics teicoplanin and vancomycin are currently the mainstay of treatment of infections with MRSA. ${ }^{1}$ However, strains of MRSA have emerged that exhibit higher than usual minimum inhibitory concentration values for these antibiotics: glycopeptide-intermediate $S$ aureus (GISA), or vancomycin resistant $S$ aureus (VISA). ${ }^{4}$ Although not fully glycopeptide resistant, infections with these isolates often respond poorly to treatment with these agents. Fortunately only a small number of infections with these bacteria have been reported so far. Nevertheless, they present a considerable threat for the future.

MRSA has been considered to be less of a problem in children, and indeed it is sometimes suggested by non-paediatric microbiologists that children may be less susceptible to colonisation or infection with MRSA. However, this seems unlikely, given the ubiquity of $S$ aureus as a childhood pathogen. It is much more likely that the lower incidence of MRSA in children relates to demographic and epidemiological differences. A relatively small proportion of children receive in-patient hospital treatment, which is the most important risk factor for acquisition of MRSA. ${ }^{1}$ Paediatric units tend to be relatively independent of adult services, and to have better provision of isolation facilities, so that even in hospitals with a high prevalence of MRSA it is possible for paediatric services to be relatively unaffected. ${ }^{5}$

\section{"There is an increasing incidence of healthcare associated infections with MRSA in children with underlying conditions predisposing to infection with $S$ aureus"}

However, the situation in children may be changing. There was a recent report in this journal of an increasing incidence of MRSA in children in Leeds with cystic fibrosis, ${ }^{6}$ and in this issue, Khairulddin and colleagues ${ }^{7}$ report that the proportion of bacteraemias with $S$ aureus in children in England and Wales that were due to MRSA increased from $0.9 \%$ to $13.1 \%$ between 1990 and 2000 . Also, Arkwright and colleagues ${ }^{8}$ have recently reported an age related increase in MRSA prevalence in children in Manchester with atopic dermatitis. Neonatal units are another area of concern, with several reported MRSA outbreaks that have been difficult to control and associated with considerable morbidity. ${ }^{90}$ What all of these studies point to is an increasing incidence of healthcare associated infections with MRSA in children with underlying conditions predisposing to infection with $S$ aureus. At the same time, recent data from the USA indicate that MRSA accounts for up to $60 \%$ of community acquired infections with $S$ aureus presenting to hospitals. ${ }^{11-15}$ Many of these cases occurred in children with few or no risk factors for acquisition of MRSA, suggesting that MRSA is circulating among children in those communities. $^{11} 121415$

The emergence and spread of MRSA in children is of considerable concern, because $S$ aureus is a major paediatric pathogen, both in hospitals and in the community. In hospitals, aside from the fact that infections with MRSA are expensive and inconvenient to treat, MRSA tends to occur as an additional pathogen, rather than replacing methicillin sensitive $S$ aureus (MSSA). ${ }^{1}$ Thus when MRSA becomes established in a hospital, the overall burden of health care associated infections tends to increase. The occurrence of MRSA among children in the community could mean that common childhood cutaneous infections such as impetigo would begin to present a real therapeutic challenge, with few, if any, options for oral or topical therapy. ${ }^{16}$

\section{"There should still be an opportunity to halt, and even reverse, the current increase in MRSA in children"}

Recent data on MRSA in children suggest that paediatrics may be where adult practice was in the mid 1990s. If that is so, then there should still be an opportunity to halt, and even reverse, the current increase in MRSA in children. First, we need more information on the current extent of the problem. 
The studies referred to earlier in this commentary point to an increasing incidence of MRSA in children having hospital contact in the UK. We can take some encouragement from the fact that the incidence of MRSA bacteraemia in the specialist Children's Hospital Trusts in England is low, ${ }^{17}$ but there are no similar data for neonatal or paediatric units in other Trusts, which provide the bulk of children's services. We have even less information on the occurrence of MRSA in the community in the UK, although most of us will have anecdotal experience of seeing cases where there are no apparent risk factors.

Second, appropriate measures for control of MRSA in children must be considered. Strenuous efforts to ascertain and eliminate possible sources of MRSA, including actively seeking and treating carriers, have repeatedly been shown to be effective in preventing spread of MRSA. ${ }^{15}$ Guidelines produced jointly by the British Society for Antimicrobial Chemotherapy, the Hospital Infection Society, and the Infection Control Nurses Association give comprehensive advice on the investigation of cases of MRSA. ${ }^{1}$ While many of the recommendations are applicable to children, there is no special consideration of paediatrics. Non-neonatal paediatrics is defined as a low risk specialty, the implication being that in hospitals where MRSA is endemic, pursuance of MRSA positive children may need to be less vigorous. This is indeed likely to be the case in many hospitals, where infection control teams are stretched and have no special interest in paediatrics. While guidance is provided on staff screening in response to cases of MRSA in patients, there is no reference to pre-emptive screening of newly appointed clinical staff. However, in this author's experience, nosocomially acquired MRSA in children is often related to new staff carrying MRSA acquired at other hospitals. As a result, all new clinical staff at Birmingham Children's Hospital in specialties such as neonatal and paediatric intensive care, neonatal surgery, and cardiac services are routinely screened for MRSA. The guidelines also do not cover specific paediatric issues such as education and play services, toys, and the management of family members of children with MRSA, who may themselves become transiently or permanently colonised, and who may be using hospital kitchen facilities, or occupying hospital accommodation.

As Khairulddin and colleagues ${ }^{7}$ point out, there is an urgent need for a national review of MRSA in children, both to establish the extent of the current problem, and to implement infection control measures that can better control MRSA in neonatology and paediatrics.

Arch Dis Child 2004:89:297-298.

doi: 10.1136/adc.2003.045534

Correspondence to: Dr J W Gray, Department of Microbiology, Birmingham Children's Hospital, Steelhouse Lane, Birmingham B4 6NH, UK; jim.gray@bch.nhs.uk

\section{REFERENCES}

1 Working Party Report. Revised guidelines for the control of methicillin-resistant Staphylococcus aureus infection in hospitals. $J$ Hosp Infect 1998;39:253-90.

2 Anon. Staphylococcus aureus bacteraemia laboratory reports and methicillin susceptibility: England and Wales, 1992-2002. http:// hpa.org.uk/infections/topics_az/staphylo/ lab_data_staphyl.htm.

3 Anon Staphylococcus aureus bacteraemia: England, Wales and Northern Ireland, April to June 2003. CDR Weekly 2003;13:1-5.

4 Linares J. The VISA/GISA problem: therapeutic implications. Clin Microbiol Infect 2001;7/suppl 4):8-15.

5 Barakate MS, Yang YX, Foo SH, et al. An epidemiological survey of methicillin-resistant
Staphylococcus aureus in a tertiary referral hospital. J Hosp Infect 2000;44:19-26.

6 Miall LS, McGinley NT, Brownlee KG, et al. Methicillin-resistant Staphylococcus aureus (MRSA) infection in cystic fibrosis. Arch Dis Child 2001;84:160-2.

7 Khairulddin N, Bishop L, Lamagni TL, et al. Emergence of methicillin resistant Staphylococcus aureus (MRSA) bacteraemia among children in England and Wales, 1990-2001. Arch Dis Child 2004;89:378-9.

8 Arkwright PD, Daniel TO, Sanyal D, et al. Agerelated prevalence and antibiotic resistance of pathogenic staphylococci and streptococci in children with infected atopic dermatitis at a singlespecialty center. Arch Dermatol 2002; 138:939-41.

9 Andersen BM, Lindemann R, Bergh K, et al. Spread of methicillin-resistant Staphylococcus aureus in a neonatal intensive unit associated with understaffing, overcrowding and mixing of patients. J Hosp Infect 2002:50:18-24.

10 Nambiar S, Herwaldt LA, Singh N. Outbreak of invasive disease caused by methicillin-resistant Staphylococcus aureus in neonates and prevalence in the neonatal intensive care unit. Pediatr Crit Care Med 2003:4:220-6.

11 Campbell AL, Bryant KA, Stover B, et al Epidemiology of methicillin-resistant Staphylococcus aureus in a children's hospital. Infect Control Hosp Epidemiol 2003:24:427-30.

12 Hussain FM, Boyle-Vavra S, Bethel D, et al. Current trends in community-acquired methicillinresistant Staphylococcus aureus at a tertiary care pediatric facility. Pediatr Infect Dis $J$ 2000; 19: 1163-6.

13 Johnigan RH, Pereira KD, Poole MD. Communityacquired methicillin-resistant Staphylococcus aureus in children and adolescents: changing trends. Arch Otolaryngol Head Neck Surg 2003;129:1049-52

14 Nakamura M, Rohling KL, Shashaty $M$, et al. Prevalence of methicillin-resistant Staphylococcus aureus nasal carriage in the community paediatric population. Pediatr Infect Dis $J$ 2002;21:917-22.

15 Sattler CA, Mason EO Jr, Kaplan SL. Prospective comparison of risk factors and demographic and clinical characteristics of community-acquired, methicilln-resistant, versus methicillin-susceptible Staphylococcus aureus infection in children. Pediatr Infect Dis J 2002;21:910-17.

16 Eady EA, Cove JH. Staphylococcal resistance revisited: community-acquired methicillin resistant Staphylococcus aureus - an emerging problem for the management of skin and soft tissue infections. Curr Opin Infect Dis 2003;16:103-24.

17 Anon. First report of the Department of Health's mandatory MRSA bacteraemia surveillance scheme in acute NHS Trusts in England: April to September 2001. CDR Weekly 2002; 12:1-15.

\section{Intravenous immunoglobulin for cystic fibrosis lung disease}

\section{R L Smyth}

\section{Commentary on the paper by Balfour-Lynn et al}

$\mathrm{n}$ a retrospective review published in this issue, ${ }^{1}$ Balfour-Lynn and colleagues describe 16 children with cystic fibrosis (CF) who appeared to show clinical improvement following regular infusions of intravenous immunoglobulin (IVIG). They have not described any criteria for the commencement of treatment, but the majority of children had previously been diagnosed with allergic bronchopulmonary aspergillosis (ABPA). An analysis of efficacy compared lung function and other concomitant treatments before starting therapy and after courses of therapy, the number of which varied considerably between patients. This treatment was associated with a reduction in the doses of oral and inhaled steroids. There was some improvement in forced vital capacity, but no difference in forced expiratory volume in one second.

Clinical practice has been likened to an experiment, where a patient presents with a problem, treatment is initiated, and the results of treatment are later assessed and conclusions drawn about whether or not the treatment is effective. There are a number of factors 
which may act to bias these conclusions. $^{2}$ Firstly the patient may have improved anyway. Secondly, new treatments are more likely to be initiated at a time when, as part of the normal fluctuation in the illness, symptoms, signs, and other measures have reached temporary extreme values which, will "regress towards the mean" when next measured. Any treatment started between the time points will appear to be effective. Thirdly, there is a well described placebo effect and finally, in the absence of blinding, the expectations of the patients and/or clinicians may bias the assessment of whether or not the treatment has been effective. This is particularly the case when outcome measures are based on clinical decisions, such as use of other concomitant therapies.

The $\mathrm{n}$ of 1 trial, in which randomised, double blind, multiple crossover comparisons of active and placebo treatments are conducted in an individual patient, ${ }^{2}$ has been developed to limit the biases inherent in uncontrolled therapeutic trials. There are a number of situations in which $n$ of 1 trials may be considered. For example, as proposed by these authors, there are situations where clinical trials have not been conducted or may not be feasible. ${ }^{2}$ It has also been suggested that they can be used in the early phases of drug development, to assist in the planning of subsequent large clinical trials. ${ }^{3}$ Finally, where a treatment has been shown in clinical trials to have an overall benefit, but there is considerable individual variation in response, $\mathrm{n}$ of $\mathrm{l}$ trials can identify which patients are likely to derive benefit. To address the last of these questions, $\mathrm{n}$ of 1 trials of recombinant DNase have been successfully performed in CF patients. ${ }^{45}$ Prerequisites for conducting $\mathrm{n}$ of $\mathrm{l}$ trials are that the underlying condition should be stable over time, the treatment should exert its effect over a short time, and this effect should stop quickly once the treatment is withdrawn. ${ }^{6}$

Uncontrolled clinical trials, such as those described in the paper by BalfourLynn and colleagues, ${ }^{1}$ have important limitations. Is the alternative, suggested by these authors, of undertaking $\mathrm{n}$ of $\mathrm{l}$ trials of intravenous immunoglobulin in "carefully selected patients" with CF, appropriate? It is not clear from this report whether the patients with severe CF lung disease, who were included, represent a group whose lung disease would be stable, at least over the duration of an $n$ of 1 trial. It is implied in the report, that the response to treatment may be cumulative and therefore multiple crossovers of one month's active treatment and one month's placebo treatment may not enable an adequate assessment. Similarly the observation that improvement was maintained after stopping treatment or indeed that the illness was "switched off" completely suggest that this therapy is not amenable to assessment by $\mathrm{n}$ of 1 trials. As others have observed, ${ }^{4}$ setting up and complying with the protocol in an $n$ of 1 trial requires considerable time and commitment on behalf of patients and clinicians. The authors of this report have suggested that a randomised controlled trial would not be ethical because of the need to administer placebo in the control group, but this would also the case in an $\mathrm{n}$ of 1 trial.

What then is the appropriate way to assess effectiveness of treatments in a small, heterogeneous group of patients such as this? These authors have alluded to the problems of conducting clinical trials in a rare disease such as CF, particularly where only a small subset of patients would be eligible. The majority of this group had a previous diagnosis of ABPA and in a proportion it was judged to be still active at the initiation of IVIG. It is possible that the clinical benefits were due to a modulation of this complication. ABPA is being increasingly recognised in $\mathrm{CF}$ and a search of The Cochrane Library ${ }^{7}$ has revealed no clinical trials of any therapy for this condition in CF. It is likely that there are sufficient CF patients with ABPA nationally to provide an adequate sample size for a UK-wide clinical trial of such therapies, and this could be facilitated by assessing numbers from the UK Cystic Fibrosis database.

I would suggest that the way to approach evaluation of treatment in such challenging clinical settings is to first consider randomised controlled trials. The exponential increase of randomised controlled trials in $\mathrm{CF}$ in the past 30 years suggests that some of the feasibility issues are being overcome. ${ }^{8}$ This may involve a certain amount of "thinking outside the box" and considering carefully what the components of each complex clinical problem might be. $\mathrm{N}$ of 1 trials may certainly have a place, but careful thought should be given to whether the condition and the treatment are amenable to assessment in this way. Observational studies may be helpful, particularly in the assessment of adverse effects. ${ }^{9}$ Case reports such as this ${ }^{1}$ are valuable, but only insofar as they generate hypotheses which lead to definitive studies rather than on their own providing conclusive evidence on which to base treatment.

Arch Dis Child 2004;89:298-299.

doi: 10.1136/adc.2003.040295

Correspondence to: Prof. R L SmythBrough Professor of Paediatric Medicine, University of Liverpool, Institute of Child Health, Alder Hey Children's Hospital, Liverpool L12 2AP, UK; r.l. smyth@liv.ac.uk

\section{REFERENCES}

1 Balfour-Lynn IM, Mohan U, Bush A, et al. Intravenous immunoglobulin for cystic fibrosis lung disease: a case series of 16 children. Arch Dis Child 2004;89:315-9.

2 Guyatt G, Sackett D, Taylor DW, et al. Determining optimal therapy-randomized trials in individual patients. N Engl J Med 1986;314:889-92.

3 Guyatt GH, Heyting A, Jaeschke R, et al. N of 1 randomized trials for investigating new drugs. Control Clin Trials 1990;11:88-100.

4 Bollert FG, Paton JY, Marshall TG, et al. Recombinant DNase in cystic fibrosis: a protocol for targeted introduction through n-of- 1 trials. Scottish Cystic Fibrosis Group. Eur Respir J 1999;13:107-13.

5 Webb S, Tansey P, Brown H, et al. Placebocontrolled n-of-1 trials in cystic fibrosis. Eur Respir J 1999;14:993.

6 Mahon J, Laupacis A, Donner A, et al. Randomised study of $\mathrm{n}$ of 1 trials versus standard practice. BMJ 1996;312:1069-74.

7 The Cochrane Library, Issue 1. Chichester UK: John Wiley \& Sons, 2004.

8 Cheng K, Smyth RL, Motley J, et al. Randomized controlled trials in cystic fibrosis (1966-1997) categorized by time, design, and intervention. Pediatr Pulmonol 2000;29:1-7.

9 Elphick HE, Tan A, Ashby D, et al. Systematic reviews and lifelong diseases. BMJ 2002;325:381-4. 